\title{
OWA-Based Extensions of the Chamberlin-Courant Rule
}

\author{
Edith Elkind ${ }^{1}$ and Anisse Ismaili ${ }^{2}$ \\ 1 Department of Computer Science, University of Oxford, United Kingdom \\ elkindecs.ox.ac.uk \\ ${ }^{2}$ Université Pierre et Marie Curie, Univ Paris 06, UMR 7606, LIP6, F-75005, Paris, France \\ anisse.ismaili@lip6.fr
}

\begin{abstract}
Given a set of voters $V$, a set of candidates $C$, and voters' preferences over the candidates, multiwinner voting rules output a fixed-size subset of candidates (committee). Under the Chamberlin-Courant multiwinner voting rule, one fixes a scoring vector of length $|C|$, and each voter's 'utility' for a given committee is defined to be the score that she assigns to her most preferred candidate in that committee; the goal is then to find a committee that maximizes the joint utility of all voters. The joint utility is typically identified either with the sum of all voters' utilities or with the utility of the least satisfied voter, resulting in, respectively, the utilitarian and the egalitarian variant of the Chamberlin-Courant's rule. For both of these cases, the problem of computing an optimal committee is NP-hard for general preferences, but becomes polynomial-time solvable if voters' preferences are single-peaked or single-crossing. In this paper, we propose a family of multiwinner voting rules that are based on the concept of ordered weighted average (OWA) and smoothly interpolate between the egalitarian and the utilitarian variants of the Chamberlin-Courant rule. We show that under moderate constraints on the weight vector we can recover many of the algorithmic results known for the egalitarian and the utilitarian version of Chamberlin-Courant's rule in this more general setting.
\end{abstract}

\section{Introduction}

Local organizers of a conference have to make an important decision: the catering company that was contracted to provide snacks for the coffee break has a menu consisting on $30+$ items, but for each coffee break one is allowed to pick at most 6 item types (cookies, pastries, finger sandwiches, etc.). If the organizers have a good estimate of the participants' preferences, they may want to ensure that each participant likes at least one of the items that are served in the coffee break. Note that participants' preferences over items other than their top item (among the ones ordered) are not important here: if Alice likes chocolate cookies, but hates cucumber sandwiches, the organizers can order both, and Alice can simply ignore the sandwiches. However, if the participants' 
preferences are very diverse, it may be impossible to ensure that everyone's favorite items are ordered; the organizers may then have to choose between providing $95 \%$ of the attendants with items they rank highly and making alternative arrangements for the remaining picky eaters, or ordering items that noone really likes, but everyone considers acceptable.

A similar issue arises when selecting the board of a large organization, such as, e.g., IFAAMAS (the non-profit foundation that runs the AAMAS conference): it is desirable to ensure that most members of the organization feel that they are represented by the board, but it may be difficult to ensure that this criterion is satisfied for very small minorities.

In their pioneering work, Chamberlin and Courant [10] described a voting procedure for selecting a committee, which is driven by such considerations. Their primary motivation was to provide a method for selecting a representative parliament, but, as illustrated above, this approach has a wider application domain (see, e.g., the recent papers of Elkind et al. [13] and Skowron et al. [25], which discuss a variety of scenarios where the Chamberlin-Courant rule and its variants may be useful). In more detail, Chamberlin and Courant consider the setting where a group of voters has to select a fixed-size committee from the available candidates, and each voter ranks all candidates from best to worst. A voter's satisfaction (utility) from a given committee is determined by the rank of her most preferred candidate in that committee within her preference order, and the goal is to select a committee that maximizes the sum of voters' satisfactions (we provide a formal definition in Section 2). A Rawlsian variant of this method was later proposed by Betzler et al. [3]: instead of maximizing the sum of voters' satisfactions, they focus on maximizing the satisfaction of the least happy voter; the subsequent literature refers to the original variant of the Chamberlin-Courant rule as the utilitarian Chamberlin-Courant rule (U-CC), and the Betzler et al.'s variant as the egalitarian Chamberlin-Courant rule (E-CC).

In this paper, we propose a family of committee selection rules that smoothly interpolate between $\mathrm{U}-\mathrm{CC}$ and $\mathrm{E}-\mathrm{CC}$ by using the concept of ordered weighted averages $(O W A)$. A rule in this family is determined by a weight vector $\boldsymbol{w}=\left(w_{1}, \ldots, w_{n}\right)$ where $n$ is the number of voters; the entries of this vector are non-negative reals that sum up to 1 . These weights are used when aggregating the voters' satisfaction: the total satisfaction is a weighted sum, where the satisfaction of the least happy voter is taken with weight $w_{1}$, the satisfaction of the second least happy voter is taken with weight $w_{2}$, etc. This framework captures both U-CC (by setting $\boldsymbol{w}=\left(\frac{1}{n}, \ldots, \frac{1}{n}\right)$ ) and E-CC (by setting $\boldsymbol{w}=(1,0, \ldots, 0))$, and allows us to trade off the utilitarian welfare and egalitarian objectives in a variety of ways. We describe a few such tradeoffs below.

- We can choose to ignore $d$ most unhappy voters (e.g., because we can make alternative provisions for them) and maximize the minimum utility among the remaining voters, by setting the weight vector to be $\boldsymbol{w}=(0, \ldots, 0,1,0, \ldots, 0)$, where the only 1 appears in position $d+1$. We denote this rule by $\mathrm{E}^{-d}-\mathrm{CC}$.

- As in the previous case, we can ignore $d$ most unhappy voters and maximize the sum of utilities of the remaining voters; this corresponds to the weight vector $\boldsymbol{w}=$ $\left(0, \ldots, 0, \frac{1}{n-d}, \ldots, \frac{1}{n-d}\right)$, where 0 s appear in the first $d$ positions. We denote this rule by $\mathrm{U}^{-d_{-}} \mathrm{CC}$. 
- We can take the opposite approach and focus on $d$ least happy voters, aiming to maximize the sum of their utilities. We denote the resulting rule, which corresponds to the weight vector $\boldsymbol{w}=\left(\frac{1}{d}, \ldots, \frac{1}{d}, 0, \ldots, 0\right)$, by $\mathrm{U}^{d}-\mathrm{CC}$. Note that $\mathrm{U}^{1}-\mathrm{CC}$ is simply E-CC, and $\mathrm{U}^{n}-\mathrm{CC}$ is U-CC.

- We can consider a refinement of E-CC, where we first maximize the utility of the least happy voter, then, among all committees that accomplish this, we select the ones that maximize the utility of the second least happy voter, etc; if voters' utilities take values in the range $[0, K]$, this rule, which we call Lex-E-CC, can be implemented by setting

$$
\boldsymbol{w}=\left(\alpha(K+1)^{n-1}, \alpha(K+1)^{n-2}, \ldots, \alpha\right),
$$

where $\alpha=\frac{K}{(K+1)^{n}-1}$ is the normalization factor. The appeal of the resulting rule is that it provides a principled refinement of E-CC, which can easily be seen to be rather indecisive.

- An alternative refinement of E-CC is to break ties based on the utilitarian social welfare, i.e., choose a committee that maximizes the sum of voters' utilities among all committees that maximize the utility of the least satisfied voter. We denote this rule by EU-CC. It is captured by the weight vector

$$
\boldsymbol{w}=\left(\frac{n K+1}{n(K+1)}, \frac{1}{n(K+1)}, \ldots, \frac{1}{n(K+1)}\right) ;
$$

again, we assume that the voters' utilities lie in the range $[0 ; K]$.

Having defined this family of committee selection rules, we then focus on their computational complexity. Both E-CC and U-CC are known to be computationally hard for general preferences, but admit efficient algorithms for restricted preference domains (see Section 1.1 for an overview of the related literature) or if the number of voters/candidates is small. We show that most of these positive results extend to many families of weight vectors, including, in particular, EU-CC, $\mathrm{U}^{-d_{-}} \mathrm{CC}, \mathrm{E}^{-d_{-}} \mathrm{CC}$, and $\mathrm{U}^{d}$-CC (for some of these results, we also need a mild restriction on the voters' score functions). This means that the additional flexibility obtained by varying the weights does not necessarily imply a substantial penalty in terms of computation time. However, there are families of weight vectors (including, notably, Lex-E-CC) that cannot be captured by our approach. We conclude the paper by discussing the limitations of our techniques and outlining directions for future work.

\subsection{Related Work}

The committee selection rules considered in this paper build on the original model of Chamberlin and Courant [10], who put forward what we call the utilitarian version of this rule; its egalitarian version was subsequently proposed by Betzler et al. [3]. A modification of the original Chamberlin-Courant rule where each member of the selected committee represents approximately the same number of voters was subsequently suggested by Monroe [21]. Our general framework extends to the Monroe rule; however, this rule appears to be more challenging from the computational perspective [3,27], and 
therefore we leave the algorithmic analysis of its OWA-based extensions as a topic for future work.

The study of computational aspects of the Chamberlin-Courant rule was initiated by Procaccia et al. [24], who showed that computing U-CC is NP-hard for approval-like utility functions; Lu and Boutilier [20] extend this result to Borda-like utility functions. Betzler et al. [3] demonstrate NP-hardness of E-CC and study fixed-parameter complexity of both E-CC and U-CC; they demonstrate that both of these rules become polynomial-time computable when voters' preferences are single-peaked. Cornaz et al. [11] extend these results to elections with bounded single-peaked width. Skowron et al. [27] show that both E-CC and U-CC remain easy when voters' preferences are single-crossing, or have bounded single-crossing width. Yu et al. [32] analyze the complexity of E-CC and U-CC when voters' preferences are single-peaked on a tree. Lu and Boutilier [20] and Skowron et al. [26] propose approximation algorithms for U-CC and E-CC.

Another extension of the Chamberlin-Courant rule that is based on OWAs was proposed by Skowron et al. [25]. However, their approach is orthogonal to ours: Skowron et al. consider the possibility that the voter cares not just about her most preferred candidate in the committee, but also derives additional utility from her second most preferred committee member, etc. (in our coffee break example, a conference participant may want to try several different snacks). Thus, while we consider weight vectors that have an entry for each voter, the weight vectors in the work of Skowron et al. have $m$ entries, where $m$ is the number of candidates.

Amanatidis et al. [1] also define a family of voting rules that use OWAs to aggregate voters' preferences; however, in contrast with our work, they consider the setting where voters' preferences are dichotomous. That is, the rules in this family interpolate between Minisum approval voting and Minimax approval voting (see [6, 19,9] for the definitions and a discussion of these rules). They prove that most of the rules constructed in this manner are NP-hard, but identify a number of tractable/approximable special cases.

The ordered weighted averaging aggregation operators were introduced in multicriteria decision making by [29] and applied in multiple contexts [23, 30, 31]. Goldsmith et al. [17] recently proposed using OWAs in the context of voting; however, in contrast with our work, they focus on single-winner settings. OWAs are typically used to accomplish fairness. In the context of multiagent assignment, fairness is formalized as monotonicity with Pareto dominance and Pigou-Dalton transfers [16], and is therefore associated with weight vectors that satisfy $w_{i}>w_{i+1}$. However, as argued above, in the context of committee elections, weight vectors that do not satisfy these inequalities (such as the ones used by $\mathrm{U}^{-d_{-}} \mathrm{CC}$ and $\mathrm{E}^{-d_{-}} \mathrm{CC}$ ) may still be useful.

\section{Preliminaries}

For $a, b \in \mathbb{N}$, let $\llbracket a, b \rrbracket=\{u \in \mathbb{N} \mid a \leq u \leq b\}$; $a, b \rrbracket=\emptyset$ if $b<a$. Similarly, let $\rrbracket a, b \llbracket=\llbracket a, b \rrbracket \backslash\{a, b\}$. The shorthand for $\llbracket 1, b \rrbracket$ is $\llbracket b \rrbracket$.

An election $E=(C, V)$ is defined by a set of candidates $C=\left\{c_{1}, \ldots, c_{m}\right\}$ and a set of voters $V=\llbracket 1, n \rrbracket$. Each voter $i \in V$ is associated with a preference order $\succ_{i}$, which is a linear order over the candidates. Given $c, c^{\prime} \in C$, we write $c \succ_{i} c^{\prime}$ 
to denote that voter $i$ prefers $c$ to $c^{\prime}$. For succinctness, we may sometimes describe the preference order of voter $i$ by listing all candidates from her most preferred one to her least preferred one and suppressing $\succ_{i}$; for instance $a b c$ is an abbreviation for $a \succ_{i} b \succ_{i} c$. Let top $(i) \in C$ denote voter $i$ 's most preferred candidate: $\operatorname{top}(i) \succ_{i} c$ for all $c \in C \backslash\{\operatorname{top}(i)\}$. The individual score, or utility, of voter $i$ is a function $s_{i}: C \rightarrow \mathbb{N}$ such that

$$
\text { for all } c, c^{\prime} \in C \quad c \succ_{i} c^{\prime} \quad \text { implies } \quad s_{i}(c) \geq s_{i}\left(c^{\prime}\right) \text {. }
$$

We let $S c r_{C, V}=\left\{s_{i}(c) \mid i \in V, c \in C\right\}$, and set $R_{C, V}=\max _{s \in S c r_{C, V}} s$; we omit $(C, V)$ from the notation when these sets are clear from the context.

We consider the setting where the goal is to select a committee of size $\kappa$. Following the ideas of Chamberlin and Courant [10], we assume that the satisfaction that a voter derives from a committee is determined by her score for her most preferred candidate in that committee. Thus, we extend the individual score function to committees by setting

$$
s_{i}(S)=\max _{c \in S}\left\{s_{i}(c)\right\} \quad \text { for each } S \subseteq C \text { with }|S|=\kappa .
$$

Existing work considers two methods of extending individual scores to group scores: the utilitarian score, which was proposed in the original work of Chamberlin and Courant [10], and the egalitarian score, suggested by Betzler et al. [3]. The utilitarian and egalitarian scores $s_{U}$ and $s_{E}$ of a committee $S \subseteq C,|S|=\kappa$, are defined by, respectively,

$$
s_{U}(S)=\sum_{i \in V} s_{i}(S) \quad \text { and } \quad s_{E}(S)=\min _{i \in V}\left\{s_{i}(S)\right\}
$$

The utilitarian $\mathrm{CC}$ rule $(\mathrm{U}-\mathrm{CC})$ and the egalitarian $\mathrm{CC}$ rule $(\mathrm{E}-\mathrm{CC})$ output a $\kappa$-sized committee that maximizes the corresponding score (breaking ties arbitrarily).

\section{Our Model}

We put forward a family of voting rules that aggregate individual scores using ordered weighted averages. A rule in this family is associated with a weight vector $\boldsymbol{w}=$ $\left(w_{1}, \ldots, w_{n}\right) \in \mathbb{R}^{n}$ such that $w_{i} \geq 0$ for all $i \in V$ and $\sum_{i \in V} w_{i}=1$. Given a committee $S \subseteq C$, let $\sigma$ be a permutation of $V$ that satisfies

$$
s_{\sigma(1)}(S) \leq \ldots \leq s_{\sigma(i)}(S) \leq \ldots \leq s_{\sigma(n)}(S)
$$

i.e., $\sigma$ orders the voters according to their score in non-decreasing order. Given an election $E=(C, V)$ and a committee size $\kappa$, the rule $\boldsymbol{w}$-CC outputs a committee of size $\kappa$ with the highest $\boldsymbol{w}$-CC score, which is computed as

$$
s_{\boldsymbol{w}}(S)=\sum_{i \in V} w_{i} s_{\sigma(i)}(S)
$$

Note that for $\boldsymbol{w}=\left(\frac{1}{n}, \ldots, \frac{1}{n}\right)$ we obtain the U-CC rule and for $\boldsymbol{w}=(1,0, \ldots, 0)$ we obtain the E-CC rule. 
Example 1. Suppose that $\kappa=2$, and consider an election over the set of candidates $C=\{a, b, c, d, e, f\}$ and 6 voters with the following preferences:

$$
\text { acebdf: } 3 \text { voters bdface: } 2 \text { voters efdbca: } 1 \text { voter. }
$$

Suppose that each voter uses the Borda score function, i.e., assigns $m-i$ points to the candidate she ranks in position $i(m=6$ in this example).

It is easy to verify that the only optimal committee with respect to U-CC is $\{a, b\}$, with a total score of 27 . Let us now consider E-CC. As no candidate is ranked in top two positions by two different groups of voters, for every committee there is at least one voter who ranks her most preferred committee member in position 3 or lower. On the other hand, there are several committees that ensure a utility of 3 to each voter: some examples are $\{a, f\},\{a, d\},\{e, b\}$, and $\{e, f\}$. E-CC can output any of these committees. In contrast, under EU-CC there is a single optimal committee, namely, $\{a, d\}$. Indeed, $\{a, d\}$ accomplishes a total score of $15+8+3=26$, while providing a utility of at least 3 to each voter. This is also the unique output of Lex-E-CC: there is one voter whose utility is 3 , two voters whose utility is 4 , and three voters whose utility is 5 , and this can easily be seen to be the best possible. The rules $\mathrm{E}^{-1}-\mathrm{CC}$ and $\mathrm{U}^{-1}-\mathrm{CC}$ necessarily output $\{a, b\}$, as this is the only committee that ensures the maximum utility to 5 voters; in contrast, $\mathrm{E}^{-2}-\mathrm{CC}$ and $\mathrm{U}^{-2}-\mathrm{CC}$ may output either $\{a, b\}$ or $\{a, e\}$, and for $\mathrm{E}^{-3}-\mathrm{CC}$ and $\mathrm{U}^{-3}-\mathrm{CC}$ any of the committees $\{a, b\},\{a, c\},\{a, d\},\{a, e\},\{a, f\}$, and $\{b, e\}$ is an acceptable answer.

\section{General Observations}

We start our algorithmic analysis of $\boldsymbol{w}$-CC rules by formalizing the respective computational problem and making a few observations about its complexity for general preferences.

To avoid dealing with representation issues for real numbers, from now on we assume that the entries of the weight vector $\boldsymbol{w}$ are non-negative rational numbers. For readability, we also make the simplifying assumption that arithmetic operations involving weights and scores can be performed in time $O(1)$; however, this assumption is not essential, and, in particular, all algorithms described in this paper still run in polynomial time if we assume that the running time of arithmetic operations is polynomial in the number of bits in the input.

We observe that, in this model, for any given weight vector $\boldsymbol{w}$, the $\boldsymbol{w}$-CC score of a given committee can be computed in polynomial time. We therefore focus on the problem of finding a winning committee, which can be formalized as follows:

WEIGHTED-CC-WINNER:

Input: an election $E=(C, V)$ with $|C|=m,|V|=n$, voters' preferences $\left(\succ_{i}\right)_{i \in V}$ and score functions $\left(s_{i}\right)_{i \in V}$ (where each $s_{i}$ is described by a list of $m$ integers), a weight vector $\boldsymbol{w}=\left(w_{1}, \ldots, w_{n}\right)$, and a committee size $\kappa$.

Output: Some committee $S \subseteq C$ of size $\kappa$ with the maximum $\boldsymbol{w}$-CC score.

Clearly, this problem is NP-hard; this follows from the fact that it generalizes the problem of finding a U-CC winning committee, which is known to be NP-hard [24,20]. 
Thus, from now on we focus on identifying tractable special cases of this problem by making additional assumption on the properties of the weight vector and/or the voters' preferences.

Few Candidates or Voters We first observe that WEIGHTED-CC-WINNER is easy if the number of candidates $m$ is small: this problem admits an algorithm whose running time is $\operatorname{poly}(n, m) 2^{m}$. The argument is similar to the one in [3]: we can go over all $\left(\begin{array}{c}m \\ \kappa\end{array}\right) \leq 2^{m}$ potential committees, evaluate the $\boldsymbol{w}$-CC score of each committee, and pick a committee with the highest score. This observation implies the following proposition (see, e.g., [22] for an introduction to fixed-parameter tractability).

Proposition 1. WeIGHTED-CC-WINNER is fixed-parameter tractable with respect to $m$.

Betzler et al. [3] also describe an algorithm that finds a U-CC winner in time poly $(n, m) n^{n}$, and therefore is useful when the number of voters is small. This algorithm proceeds by considering all ways of partitioning the $n$ voters into at most $\kappa$ groups. For each partition, it constructs a bipartite graph where the left-hand side corresponds to groups of voters, the right-hand side corresponds to candidates, and the value of an edge is the total utility that the respective group of voters derives from the respective candidate. It then finds a maximum-value matching of size $\kappa$ in this graph. However, it seems to be difficult to extend this idea to arbitrary weights: the following example illustrates that it is not clear how to define edge values in a meaningful way.

Example 2. Suppose that $\kappa=2$, and consider an election over the set of candidates $C=\{a, b, c, d\}$ and 4 voters with the following preferences:

$$
a b d c: 2 \text { voters } \quad c d b a: 1 \text { voter } \quad b a d c: 1 \text { voter }
$$

Let $\boldsymbol{w}=(.4, .3, .2, .1)$, and suppose that the score function of each voter is the Borda score function (see Example 1).

Suppose we consider a partition where the voters with preferences $a b d c$ form one group, and the other two voters form another group, and we would like to evaluate the contribution of the second group of voters to the total utility if we were to match it to candidate $d$. However, to do so, we would have to know which candidate is matched to the first group: if it is $a$, then the contribution of the second group is $.4 \cdot 2+.3 \cdot 1$, whereas if it is $c$, the contribution of the second group is $.2 \cdot 2+.1 \cdot 1$.

EU-CC rule and Generalizations We have argued that the EU-CC rule offers a useful balance between egalitarian and utilitarian welfare. We will now show that it also has computational advantages: computing EU-CC winners is no harder than computing UCC winners.

Specifically, observe that the E-CC score of any committee is an element of $\mathrm{Scr}$, and note that $|S c r| \leq n m$. Let $\Delta=n R$, where $R=\max _{s \in S c r} s$. We can now consider all elements $z \in S c r$ in decreasing order. For a given value of $z$, we modify the score functions $s_{i}(c)$ so that $s_{i}^{\prime}(c)=s_{i}(c)+\Delta$ if $s_{i}(c) \geq z$ and $s_{i}^{\prime}(c)=0$ otherwise. Note that the modified score functions remain consistent with voters' preferences. If we call an algorithm for U-CC on the resulting instance, we obtain a committee whose U-CC 
score is at least $\Delta \kappa$ if and only if the original instance admits a committee where the utility of each voter is at least $z$. Moreover, if the U-CC score of a committee with the highest U-CC score is at least $\Delta \kappa$, then any such committee has the highest U-CC score in the original election among all committees that guarantee a utility of $z$ to each voter.

Thus, by finding the largest value of $z$ for which the modified instance admits a committee with U-CC score of at least $\Delta \kappa$ and outputting a committee with the maximum U-CC score for this instance, we obtain a winning committee under EU-CC for the original instance. As voters' preferences in the modified instance are the same as in the original instance (only the scores change), this implies that EU-CC admits a polynomial-time algorithm when the voters preferences are single-crossing or singlepeaked on a line or, more broadly, on a tree with a constant number of leaves, and is fixed-parameter tractable with respect to the number of voters; this follows from the respective results of $[3,27,32]$ for $\mathrm{U}-\mathrm{CC}$.

More generally, by considering all values of $z \in S c r$, we obtain the Pareto boundary of the associated bicriteria optimization problem (where the criteria are the egalitarian and the utilitarian social welfare).

Observe that a committee that is optimal with respect to EU-CC is by definition optimal with respect to E-CC (as the former is a refinement of the latter), so in particular the argument above shows that E-CC is tractable whenever U-CC is; however, specialpurpose algorithms for E-CC may be considerably faster than the algorithm provided by our reduction (as illustrated by the results of [3]).

\section{Algorithms for Single-peaked Preferences}

An election $(C, V)$ described by a collection of preference orders $\left(\succ_{i}\right)_{i \in V}$ is said to be single-peaked if there exists an ordering $\sqsubset$ of the candidates (the left-right axis) such that for each voter $i \in V$ her preference $\succ_{i}$ increases from the left of the axis to top $(i)$ and decreases from top $(i)$ to the right of the axis: formally,

for all $a, b \in C$ s.t. $\quad a \sqsubset b \sqsubseteq \operatorname{top}(i) \quad$ or $\quad \operatorname{top}(i) \sqsubseteq b \sqsubset a \quad$ it holds that $\quad b \succ_{i} a$.

Equivalently, for each voter $i \in V$ and each $k \leq m$ the set of candidates that $i$ ranks in top $k$ positions forms a contiguous interval with respect to $\sqsubset$.

Single-peaked elections were introduced by Black [4], and are known to have a number of desirable social-choice properties as well as to admit efficient algorithms for many computational social choice problems (see, e.g., [28, 15, 7]). Intuitively, such elections arise when the society is aligned along a single axis, and voters rank the candidates according to their position on this axis.

In the rest of this section, we consider two classes of weight vectors, and show how to compute the output of $\boldsymbol{w}$-CC for weight vectors in these classes, assuming that voters' preferences are single-peaked. It will be convenient to assume that the left-right axis is given explicitly, and, moreover, that this axis is $c_{1} \sqsubset \cdots \sqsubset c_{m}$. This assumption is without loss of generality: given a collection of preferences, we can decide if it is single-peaked, and, if so, find an axis witnessing this, in polynomial time [2, 12]. 


\subsection{The $\mathrm{U}^{-d}-\mathrm{CC}$ rule}

In this section, we provide a polynomial-time algorithm for $\mathrm{U}^{-d}-\mathrm{CC}$ - the rule that maximizes the sum of utilities of all but $d$ voters (and therefore ignores the $d$ least happy voters entirely) — under single-peaked preferences. In contrast with the result of Section 5.2, which deals with a larger class of weight vectors, this algorithm works for arbitrary score functions.

Let $\theta=n-d$; recall that the weight vector $\boldsymbol{w}^{-d}$ associated with $\mathrm{U}^{-d}-\mathrm{CC}$ satisfies

$$
w_{i}^{-d}= \begin{cases}0 & \text { if } i \leq d \\ \frac{1}{\theta} & \text { if } i>d\end{cases}
$$

The key observation is that it is sufficient to maximize the sum of the scores of some $\theta$ voters, without fixing in advance the voters whose score is taken into account. Indeed, this maximization process naturally selects the $\theta$ most happy voters.

Theorem 1. For single-peaked preferences, Algorithm 1 computes a winning committee under $U^{-d}$-CC in time $O\left(\kappa m^{2} \theta^{2} n \log _{2} n\right)=O\left(m^{3} n^{3} \log _{2} n\right)$.

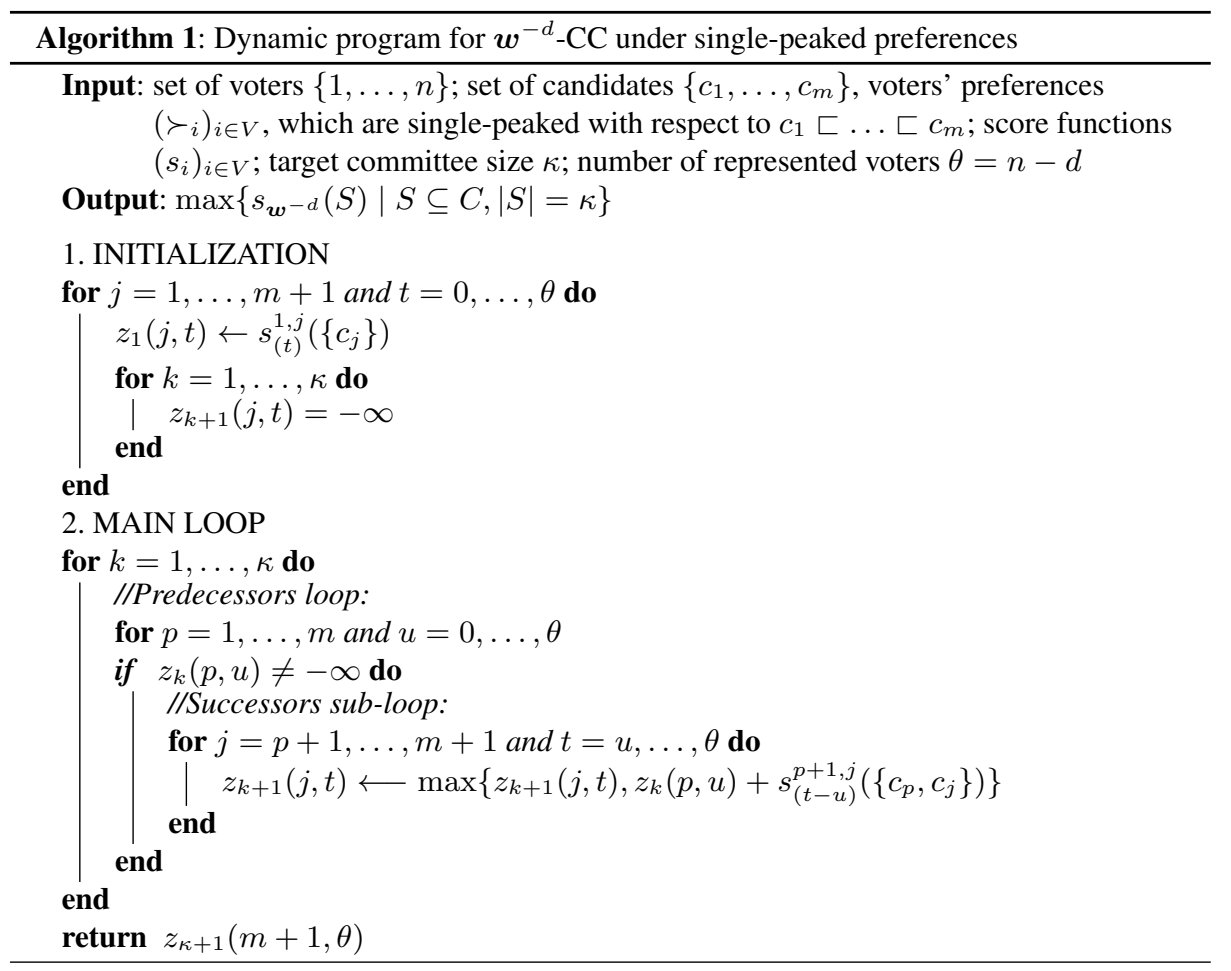


Proof. We assume without loss of generality that voters' preferences are single-peaked with respect to the axis $c_{1} \sqsubset \ldots \sqsubset c_{m}$. It will be convenient to introduce an additional candidate $c_{m+1}$ who is ranked last by each voter and such that $s_{i}\left(c_{m+1}\right)=0$ for each $i \in V$; we place this candidate to the right of $c_{m}$ on the axis so that the election remains single-peaked.

Let $V^{\ell, j}=\left\{i \in V \mid \operatorname{top}(i) \in\left\{c_{\ell}, \ldots, c_{j}\right\}\right\}$, and define

$$
s_{(t)}^{\ell, j}(S)=\max \left\{\sum_{i \in W} s_{i}(S)\left|W \subseteq V^{\ell, j},\right| W \mid=t\right\},
$$

with the convention that $\max \emptyset=-\infty$. When the set $\left\{W\left|W \subseteq V^{\ell, j},\right| W \mid=t\right\}$, is not empty, the quantity $s_{(t)}^{\ell, j}(S)$ can be computed by ordering the voters in $V^{\ell, j}$ according to the score they assign to $S$ (from the highest to the lowest), picking the first $t$ voters in this order, and summing their scores for $S$.

Now, for each $t \in\{0, \ldots, \theta\}, k \in\{1, \ldots, \kappa+1\}$, and $j \in\{k, \ldots, m+1\}$, let

$$
z_{k}(j, t)=\max \left\{s_{(t)}^{1, j}(S)\left|S \subseteq\left\{c_{1}, \ldots, c_{j}\right\}, c_{j} \in S,\right| S \mid=k\right\}
$$

The quantity $z_{k}(j, t)$ is the highest total utility that a group of $t$ voters in $V^{1, j}$ can derive from a size- $k$ subset of $\left\{c_{1}, \ldots, c_{j}\right\}$ that contains $c_{j}$. We claim that it can be computed by dynamic programming as follows: for $k=1$ we have $z_{1}(j, t)=s_{(t)}^{1, j}\left(\left\{c_{j}\right\}\right)$ for all $t \in\{0, \ldots, \theta\}, j \in\{1, \ldots, m+1\}$, and for $k>1$

$$
z_{k}(j, t)=\max _{p \in \rrbracket k, j \llbracket} \max _{u \in \llbracket 0, t \rrbracket}\left\{z_{k-1}(p, u)+s_{(t-u)}^{p+1, j}\left(\left\{c_{p}, c_{j}\right\}\right)\right\} .
$$

Indeed, to find the score of an optimal pair $(S, W)$, where $S \subseteq\left\{c_{1}, \ldots, c_{j}\right\}, c_{j} \in$ $S,|S|=k, W \subseteq V^{1, j}$, and $|W|=t$, we guess the last candidate in $S^{\prime}=S \cap$ $\left\{c_{1}, \ldots, c_{j-1}\right\}$ (let this candidate be $c_{p}$ ) and the number of voters in $W^{\prime}=W \cap V^{1, p}$ (let this number be $u$ ). We then observe that all voters in $W^{\prime}$ have their peak at or to the left of $c_{p}$ and therefore their most preferred candidate in $S$ is not $c_{j}$, whereas for each voter $i \in W \backslash W^{\prime}$ we have $\operatorname{top}(i) \in\left\{c_{p}, c_{j}\right\}$.

Algorithm 1 implements the calculation in (1) in a forward manner to increase efficiency.

It is easy to see that the $\mathrm{U}^{-d}$-CC score of a winning committee is $z_{\kappa+1}(m, \theta)$; a committee with this score can be found using standard dynamic programming techniques.

The number of variables in our dynamic program is $O(\kappa m \theta)$. To compute the value of variable $z_{k+1}(j, t)$, we need to consider $O(m \theta)$ possibilities for $p$ and $u$, and, for each of them, compute $s_{(t-u)}^{p+1, j}\left(\left\{c_{p}, c_{j}\right\}\right)$; the latter step involves sorting the voters in $V^{p+1, j}$ according to their score for $\left\{c_{p}, c_{j}\right\}$, and can therefore be implemented in time $O(n \log n)$. This implies our bound on the running time.

\section{$5.2 w$-CC for a Bounded Number of Weights}

In this section, we show that $\boldsymbol{w}$-CC admits a polynomial-time algorithm if voters' preferences are single-peaked, their score functions are polynomially bounded (i.e. 
$R=\operatorname{poly}(m, n)$ ), and the number of distinct entries in $\boldsymbol{w}$ is small. That is, we consider weight vectors of the form

$$
\boldsymbol{w}=\left(\omega_{1}, \ldots, \omega_{1}, \omega_{2}, \ldots, \omega_{2}, \ldots, \omega_{B}, \ldots, \omega_{B}\right),
$$

where $B$ is assumed to be a constant. Let $\alpha(b)$ and $\beta(b)$ be, respectively, the indices of the first and the last occurrence of $\omega_{b}$ in $\boldsymbol{w}$, and let $\gamma(b)=\beta(b)-\alpha(b)+1$. Setting $y_{b}(S)=\sum_{i=\alpha(b)}^{\beta(b)} s_{\sigma(i)}(S)$, we can rewrite the $\boldsymbol{w}$-CC score of a committee $S$ as

$$
s_{\boldsymbol{w}}(S)=\sum_{b=1}^{B} \omega_{b} \sum_{i=\alpha(b)}^{\beta(b)} s_{\sigma(i)}(S)=\sum_{b=1}^{B} w_{b} y_{b}(S) .
$$

The main idea of our algorithm is that when $B$ is a constant, we can try to guess the range of scores that will be counted with weight $\omega_{b}$, for each $b=1, \ldots, B$; for a given guess, we can use dynamic programming.

Theorem 2. When voters' preferences are single-peaked and the weight vector $\boldsymbol{w}$ is given by expression (2), a winning committee under $\boldsymbol{w}$-CC can be computed in time $\operatorname{poly}\left(n, m,(n m)^{B}, R^{B}\right)$, where $R=\max _{i \in V, c \in C} s_{i}(c)$.

Proof. By adding or subtracting a constant to all score functions, we can assume that $\min _{r \in S c r} r=1$. Let $\mathcal{R}$ be the set of all vectors $\left(r_{0}, \ldots, r_{B}\right)$ in $S c r^{B+1}$ that satisfy $1=r_{0} \leq r_{1} \leq \cdots \leq r_{B}=R$. Note that $|\mathcal{R}| \leq|S c r|^{B-1} \leq(n m)^{B-1}$; if $B$ is a constant, this quantity is polynomial in the input size.

Each vector $\boldsymbol{r} \in \mathcal{R}$ induces a partition $\Pi^{r}$ of $S c r$ into $2 B-1$ sub-ranges, some of which may be empty:

$$
\Pi^{r}=\left\{\quad \llbracket 0, r_{1} \llbracket,\left\{r_{1}\right\}, \rrbracket r_{1}, r_{2} \llbracket,\left\{r_{2}\right\}, \ldots,\left\{r_{B-1}\right\}, \rrbracket r_{B-1}, r^{*} \rrbracket\right\} .
$$

We say that a committee $S$ is $\boldsymbol{r}$-compatible if, when we order the voters according to their score for this committee from lowest to highest, then for each $b=1, \ldots, B-1$ it holds that the score of the voter in position $\beta_{b}$ is $r_{b}$.

Given an instance of WEIGHTED-CC-WINNER where the weight vector $\boldsymbol{w}$ has $B$ distinct entries, our algorithm considers all vectors in $\mathcal{R}$. For each such vector $\boldsymbol{r}$, it calls the subroutine described in Algorithm 2. It then outputs the maximum of the $|\mathcal{R}| \leq(n m)^{B}$ numbers obtained in this manner. We will now argue that for a given $\boldsymbol{r}$, Algorithm 2 returns the maximum $\boldsymbol{w}$-CC score of an $\boldsymbol{r}$-compatible committee of size $\kappa$. Since an optimal committee of size $\kappa$ is compatible with some $r \in \mathcal{R}$, this proves that our algorithm computes the maximum $\boldsymbol{w}$-CC score over all committees of size $\kappa$; a committee with this score can then be found using standard techniques.

As in the proof of Theorem 1, we add a dummy candidate $c_{m+1}$ that appears to the right of $c_{m}$ on the axis and is ranked last by all voters. Also, as in that proof, we denote by $V^{\ell, j}$ the set of voters whose top candidate is in $\left\{c_{\ell}, \ldots, c_{j}\right\}$.

Fix a vector $\boldsymbol{r} \in \mathcal{R}$ and the respective partition $\Pi^{\boldsymbol{r}}=\left\{\rho_{1}, \ldots, \rho_{2 B-1}\right\}$. For a given committee $S$, let $V(S, j, d)$ be the set of all voters in $V^{1, j}$ whose score for $S$ lies in $\rho_{d}$ : $V(S, j, d)=\left\{i \in V^{1, j} \mid s_{i}(S) \in \rho_{d}\right\}$. 
We are now ready to define the variables of our dynamic program. For each $\boldsymbol{r} \in \mathcal{R}$, each $k=1, \ldots, \kappa+1$, each $j=k, \ldots, m+1$, and each $t \in \llbracket 0, n \rrbracket^{2 B-1}$, we define a variable $Y_{k}^{\boldsymbol{r}}(j, \boldsymbol{t})$. This is a collection of vectors of length $2 B-1$ with entries in $\llbracket n R \rrbracket$; the vectors in $Y_{k}^{\boldsymbol{r}}(j, \boldsymbol{t})$ are 'realizable' combinations of scores. That is, a vector $\boldsymbol{y} \in \llbracket n R \rrbracket^{2 B-1}$ is in $Y_{k}^{\boldsymbol{r}}(j, \boldsymbol{t})$ if and only if there exists a committee $S \subseteq\left\{c_{1}, \ldots, c_{j}\right\}$ with $c_{j} \in S,|S|=k$, such that for each $\rho_{d} \in \Pi^{r}$ we have

$$
|V(S, j, d)|=t_{d}, \quad \sum_{i \in V(S, j, d)} s_{i}(S)=y_{d}
$$

We will now explain how our algorithm computes the sets $Y_{k}^{\boldsymbol{r}}(j, \boldsymbol{t})$. For $k=1$ and a fixed value of $j$, we simply compute $s_{i}(j)$ for all voters in $V^{1, j}$. This score maps each voter in $V^{1, j}$ to some range in $\Pi^{r}$; the number of voters assigned to $\rho_{d}$ (which is exactly $\left.\left|V\left(\left\{c_{j}\right\}, j, d\right)\right|\right)$ determines $t_{d}$, and the sum of their scores for $\left\{c_{j}\right\}$ determines $y_{d}$. We obtain a pair of vectors $(\boldsymbol{t}, \boldsymbol{y})$ in this manner. We then set $Y_{1}^{\boldsymbol{r}}(j, \boldsymbol{t})=\{\boldsymbol{y}\}$; for other values of $\boldsymbol{t}^{\prime}$ the set $Y_{1}^{\boldsymbol{r}}\left(j, \boldsymbol{t}^{\prime}\right)$ remains empty.

For $k>1$, just as in the proof of Theorem 1, we consider all possibilities for the predecessor-candidate $c_{p}, p \in \llbracket k-1, j-1 \rrbracket$. All voters in $V^{1, p}$ prefer $c_{p}$ to $c_{j}$, so we need to focus on voters in $V^{p+1, j}$. Since their preferences are single-peaked with respect to $\sqsubset$, their score for any committee $S$ with $S \cap\left\{c_{p}, \ldots, c_{j}\right\}=\left\{c_{p}, c_{j}\right\}$ is equal to their score for $\left\{c_{p}, c_{j}\right\}$. We compute this score, which maps every such voter to some range in $\Pi^{r}$; the number of voters mapped to $\rho_{d}$ is given by $\mid V\left(\left\{c_{p}, c_{j}\right\}, j, d\right) \backslash$ $V\left(\left\{c_{p}, c_{j}\right\}, p, d\right) \mid$. As the overall number of voters in each range is given by $\boldsymbol{t}$, we let $\boldsymbol{t}_{d}^{\prime}=\boldsymbol{t}_{d}-\left|V\left(\left\{c_{p}, c_{j}\right\}, j, d\right) \backslash V\left(\left\{c_{p}, c_{j}\right\}, p, d\right)\right|$ for each $\rho_{d} \in \Pi^{\boldsymbol{r}}$, and consider the set $Y_{k-1}^{\boldsymbol{r}}\left(p, \boldsymbol{t}^{\prime}\right)$. The elements of this set are realizable combinations of scores for $p$ and $\boldsymbol{t}^{\prime}$; by adding the scores of voters in $V^{p+1, j}$ in each range, we obtain a realizable combination of scores for $j$ and $\boldsymbol{t}$. We add the resulting score vector to $Y_{k}^{\boldsymbol{r}}(j, \boldsymbol{t})$. Algorithm 2 performs this computation in a forward manner for added efficiency.

It remains to explain how to use $Y_{k}^{\boldsymbol{r}}(j, \boldsymbol{t})$ to compute the maximum $\boldsymbol{w}$-CC score of an $\boldsymbol{r}$-compatible committee of size $\kappa$. Recall that $\gamma_{b}$ is the number of occurrences of $\omega_{b}$ in $\boldsymbol{w}$. We say that a vector $\boldsymbol{t}$ in $\llbracket 0, n \rrbracket^{2 B-1}$ is valid if $\sum_{d=1}^{2 B-1} t_{d}=n$ and for each $b=1, \ldots, B-1$ there exists a non-negative integer $\xi_{b} \leq t_{2 b}$ such that $\gamma(1)=t_{1}+\xi_{1}$, $\gamma(b)=t_{2 b-2}-\xi_{b-1}+t_{2 b-1}+\xi_{b}$ for $b>1, \gamma(B)=t_{2 B-2}-\xi_{B-1}+t_{2 B-1}$. Intuitively, we have to 'distribute' the voters whose score is $r_{b}$ so that $\xi_{b}$ of them are counted with weight $\omega_{b}$ and the remaining ones are counted with weight $\omega_{b+1}$. Clearly, one can decide in time $O(B)$ whether a given vector is valid and find the respective integers $\left(\xi_{b}\right)_{b \in \llbracket 1, B-1 \rrbracket}$.

Suppose that $\boldsymbol{t}$ is a valid vector, as witnessed by a collection of integers $\left\{\xi_{b}\right\}_{b \in \llbracket 1, B-1 \rrbracket}$. Then, given a vector $\boldsymbol{y} \in Y_{\kappa+1}^{\boldsymbol{r}}(m+1, \boldsymbol{t})$, let $z_{1}=y_{1}+\xi_{1} r_{1}, z_{b}=\left(t_{2 b-2}-\xi_{b-1}\right) r_{b-1}+$ $y_{2 b-1}+\xi_{b} r_{b}$ for $b=2, \ldots, B-1, z_{B}=\left(t_{2 B-2}-\xi_{B-1}\right) r_{B-1}+y_{2 B-1}$, and set $T(\boldsymbol{t}, \boldsymbol{y})=\sum_{b=1}^{B} \omega_{b} z_{b}$. It is not hard to see that for a fixed vector $\boldsymbol{r}$ the maximum $\boldsymbol{w}$-score of an $\boldsymbol{r}$-compatible committee of size $\kappa$ is given by

$$
\max \left\{T(\boldsymbol{t}, \boldsymbol{y}) \mid \boldsymbol{t} \text { is valid and } \boldsymbol{y} \in Y_{\kappa+1}^{\boldsymbol{r}}(m+1, \boldsymbol{t})\right\},
$$

which is exactly the quantity output by our algorithm for that choice of $\boldsymbol{r}$. 


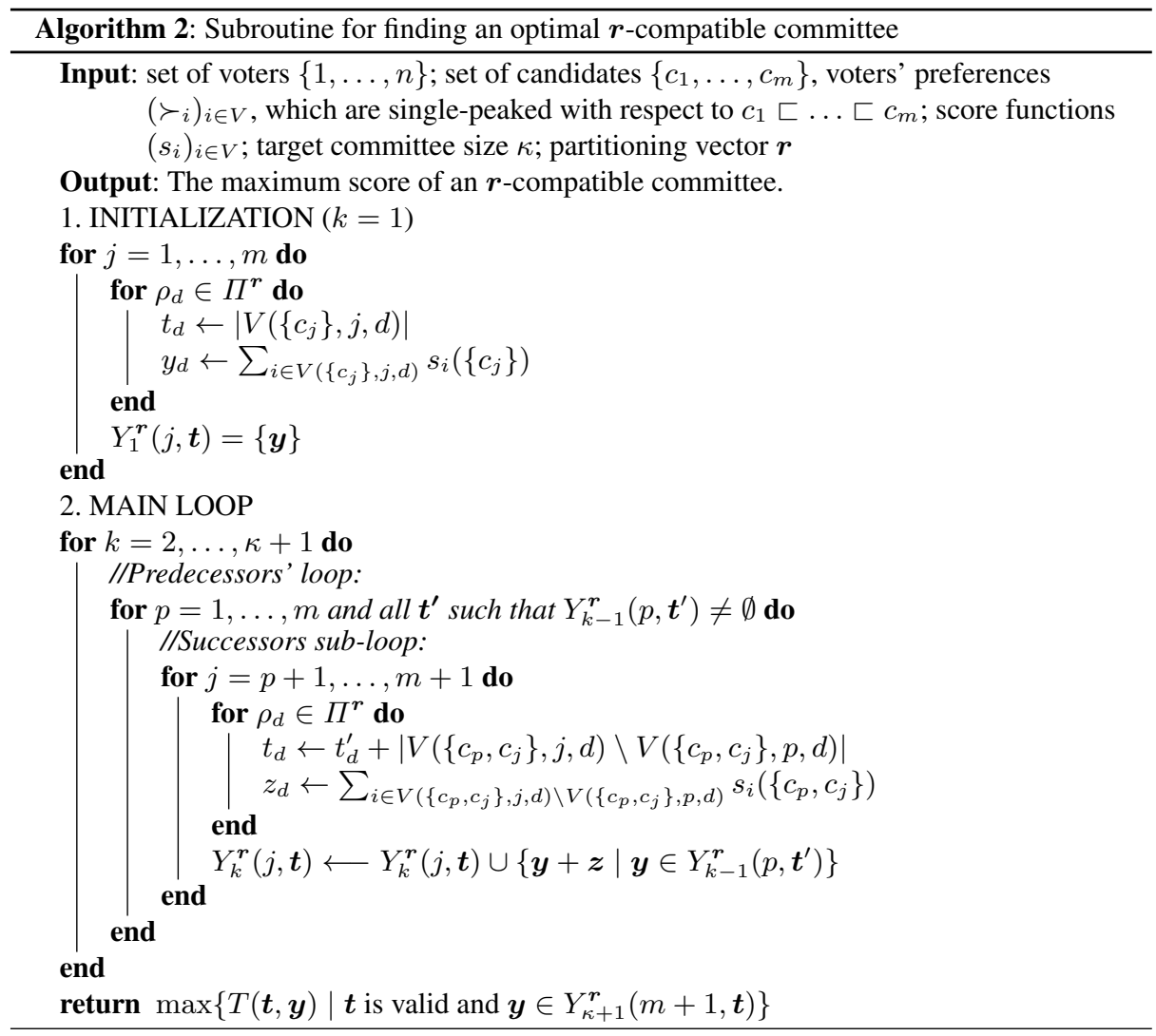

There are at most $(n m)^{B-1}(\kappa+1)(m+1)(n+1)^{2 B-1}=O\left(\kappa m\left(n^{3} m\right)^{B-1}\right)$ variables $Y_{k}^{\boldsymbol{r}}(j, \boldsymbol{t})$ : at most $(n m)^{B-1}$ choices for $\boldsymbol{r}, \kappa+1$ choices for $k, m+1$ choices for $j$, and at most $(n+1)^{2 B-1}$ choices for $\boldsymbol{t}$. Moreover, the size of each set $Y_{k}^{r}(j, \boldsymbol{t})$ can be bounded by $(n R)^{2 B-1}$; we can improve this bound to $(n R)^{B}$ by observing that if $d=2 b$ is even, then $\rho_{d}$ is the singleton $\left\{r_{b}\right\}$, so we have $y_{d}=r_{b} \cdot t_{d}$. This establishes our bound on the running time.

Remark 1. Theorem 3 relies on the assumption that the ranges of all score functions are polynomially bounded in $n$ and $m$. This assumption holds for many important score functions, such as the Borda score function; however, it is not without loss of generality. We can exhibit another class of score functions for which $\boldsymbol{w}$-CC is easy when the number of weights is bounded and the voters' preferences are single-peaked: these are score functions that can only take a constant number of values, i.e., the size of the set $S c r$ is bounded by a constant. The algorithm for this setting can be obtained by adapting the algorithm in the proof of Theorem 3; we omit the details due to space constraints. 


\section{Algorithms for Single-crossing Preferences}

In this section, we focus on elections where voters' preferences are single-crossing [18]. This is another well-known restricted preference domain that models scenarios where voters' preferences are essentially one-dimensional; however, in contrast with single-peaked preferences, which are defined in terms of an ordering of the candidates, single-crossing preferences are defined in terms of an ordering of the voters.

We start by providing a formal definition of this domain.

An election $(C, V)$ described by a collection of preference orders $\left(\succ_{i}\right)_{i \in V}$ is said to be single-crossing if there exists an ordering $\sqsubset$ of the voters such that for each pair of candidates $a, b \in C$ such that the first voter in $\sqsubset$ prefers $a$ to $b$ there exists a unique $v \in V$ such that all voters that precede $v$ in $\sqsubset$ prefer $a$ to $b$, whereas all voters that appear after $v$ in $\sqsubset$ prefer $b$ to $a$.

In other words, both the set of voters who prefer $a$ to $b$ and the set of voters who prefer $b$ to $a$ are contiguous with respect to $\sqsubset$, i.e., if we graph the positions of $a$ and $b$ in voters' preference orders, the resulting curves intersect at most once. As observed by Bredereck et al. [8], this characterization immediately suggests a polynomial-time algorithm for detecting single-crossing preferences, by reducing this question to the classic consecutive 1s problem [5] (see also [12,14]). As a consequence, we can assume without loss of generality that an ordering of voters witnessing that a given election is single-crossing is given to us as a part of the input; in fact, it will be convenient to assume that this ordering is $1 \sqsubset \cdots \sqsubset n$.

Skowron et al. [27] have recently shown that given an election with single-crossing preferences, one can solve U-CC and E-CC in polynomial time (specifically, $O\left(\kappa m n^{2}\right)$ ). The main result of this section is that the algorithm of Skowron et al. extends to $\boldsymbol{w}$-CC as long as the number of distinct weights in $\boldsymbol{w}$ is bounded by a constant and the score functions are polynomially bounded (these are the same conditions that are used to establish tractability under single-peaked preferences in Section 5).

First of all, we observe that a key structural property of optimal solutions established by Skowron et al. for U-CC holds for arbitrary weight vectors.

Given the set of voters $V=\llbracket n \rrbracket$ and a committee $S \subseteq C$, let $\Phi_{S}: V \rightarrow S$ be the function that assigns each voter $i$ to her most preferred candidate in $S: \Phi_{S}(i) \succ_{i} c_{j}$ for all $c_{j} \in S \backslash\left\{\Phi_{S}(i)\right\}$. Hence, for a candidate $c \in S$, the set of voters she represents is $\Phi_{S}^{-1}(c)$.

Lemma 1. Let $E=(C, V)$ be an election with single-crossing preferences, as witnessed by the voter order $1 \sqsubset \ldots \sqsubset n$, where the first voter's preferences are given by $c_{1} \succ_{1} c_{2} \succ_{1} \ldots \succ_{1} c_{m}$. Then for any weight vector $\boldsymbol{w}$ and any committee size $\kappa$ there exists a committee $S$ of size $\kappa$ that is optimal with respect to $\boldsymbol{w}$-CC and has the following property: for each $c_{j} \in S$ the set of voters $\Phi_{S}^{-1}\left(c_{j}\right)$ is contiguous with respect to $\sqsubset$, and if $c_{j}, c_{\ell} \in S, \Phi_{S}^{-1}\left(c_{j}\right) \neq \emptyset, \Phi_{S}^{-1}\left(c_{\ell}\right) \neq \emptyset$ and $j<\ell$ then $\Phi_{S}^{-1}\left(c_{j}\right)$ precedes $\Phi_{S}^{-1}\left(c_{\ell}\right)$ in $\sqsubset$.

Proof. The proof is a simple generalization of the proof of a similar statement in [27], and is omitted due to space constraints. 
Using this lemma and a dynamic programming approach similar to the one in the proof of Theorem 3, we obtain the following result (we omit the proof due to space constraints).

Theorem 3. When voters' preferences are single-crossing and the weight vector $\boldsymbol{w}$ has at most $B$ distinct entries, we can find a winning committee under $\boldsymbol{w}$-CC in time $\operatorname{poly}\left(n, m,(n m)^{B}, R^{B}\right)$, where $R=\max _{i \in V, c \in C} s_{i}(c)$.

\section{Conclusion}

We have described a family of voting rules for committee selection that explores a variety of ways to trade off utilitarian and egalitarian objectives. We have developed algorithms for computing the winning committees under these rules for instances where voters' preferences belong to well-known restricted domains, under mild restrictions on the weight vectors and score functions. While in this work we focused on singlepeaked and single-crossing preferences, it seems plausible that similar results can be obtained for other domains where U-CC and E-CC are known to be tractable, such as preferences that are single-peaked on trees with a bounded number of leaves, or have bounded single-peaked or single-crossing width.

However, it is not clear if our results can be extended to arbitrary weight vectors. In particular, the complexity of Lex-E-CC for single-peaked or single-crossing preferences remains an intriguing open question, and it appears that obtaining positive results for this rule would require novel algorithmic techniques.

Besides this open problem, our work suggests several other interesting research directions. One of them is considering OWA-based extensions of the Monroe rule and exploring the complexity of finding winning committees under the resulting family of rules. Another one is developing approximation algorithms for WEIGHTED-CCWINNER for arbitrary weights and voters' preferences; here the work of Skowron et al. [26] provides a useful starting point.

Acknowledgements This work was partially supported by an STSM Grant from the COST Action IC1205. The authors are grateful to the anonymous ADT referees for their useful suggestions.

\section{References}

1. Amanatidis, G., Barrot, N., Lang, J., Markakis, E., Ries, B.: Multiple referenda and multiwinner elections using hamming distances: Complexity and manipulability. In: Proceedings of the 14th International Conference on Autonomous Agents and Multiagent Systems. pp. 715-723 (2015)

2. Bartholdi, III, J., Trick, M.: Stable matching with preferences derived from a psychological model. Operation Research Letters 5(4), 165-169 (1986)

3. Betzler, N., Slinko, A., Uhlmann, J.: On the computation of fully proportional representation. Journal of Artificial Intelligence Research 47(1), 475-519 (2013)

4. Black, D.: The Theory of Committees and Elections. Cambridge University Press (1958) 
5. Booth, K., Lueker, G.: Testing for the consecutive ones property, interval graphs, and graph planarity using PQ-tree algorithms. Journal of Computer and System Sciences 13(3), 335379 (1976)

6. Brams, S., Kilgour, D.M., Sanver, R.M.: A minimax procedure for electing committees. Public Choice 132(3-4), 401-420 (2007)

7. Brandt, F., Brill, M., Hemaspaandra, E., Hemaspaandra, L.: Bypassing combinatorial protections: Polynomial-time algorithms for single-peaked electorates. Journal of Artificial Intelligence Research (2015)

8. Bredereck, R., Chen, J., Woeginger, G.: A characterization of the single-crossing domain. Social Choice and Welfare 41(4), 989-998 (2013)

9. Caragiannis, I., Kalaitzis, D., Markakis, E.: Approximation algorithms and mechanism design for minimax approval voting. In: Proceedings of the 25th AAAI Conference on Artificial Intelligence. pp. 737-742 (2010)

10. Chamberlin, B., Courant, P.: Representative deliberations and representative decisions: Proportional representation and the Borda rule. American Political Science Review 77(3), 718733 (1983)

11. Cornaz, D., Galand, L., Spanjaard, O.: Bounded single-peaked width and proportional representation. In: Proceedings of the 20th European Conference on Artificial Intelligence. pp. 270-275 (2012)

12. Doignon, J., Falmagne, J.: A polynomial time algorithm for unidimensional unfolding representations. Journal of Algorithms 16(2), 218-233 (1994)

13. Elkind, E., Faliszewski, P., Skowron, P., Slinko, A.: Properties of multiwinner voting rules. In: Proceedings of the 13th International Conference on Autonomous Agents and Multiagent Systems. pp. 53-60 (2014)

14. Elkind, E., Faliszewski, P., Slinko, A.: Clone structures in voters' preferences. In: Proceedings of the 13th ACM Conference on Electronic Commerce. pp. 496-513 (2012)

15. Faliszewski, P., Hemaspaandra, E., Hemaspaandra, L., Rothe, J.: The shield that never was: Societies with single-peaked preferences are more open to manipulation and control. Information and Computation 209(2), 89-107 (2011)

16. Golden, B., Perny, P.: Infinite order Lorenz dominance for fair multiagent optimization. In: Proceedings of the 9th International Conference on Autonomous Agents and Multiagent Systems. pp. 383-390 (2010)

17. Goldsmith, J., Lang, J., Mattei, N., Perny, P.: Voting with rank dependent scoring rules. In: Proceedings of the 28th AAAI Conference on Artificial Intelligence. pp. 698-704 (2014)

18. Grandmont, J.: Intermediate preferences and the majority rule. Econometrica 46(2), 317-330 (1978)

19. LeGrand, R., Markakis, E., Mehta, A.: Some results on approximating the minimax solution in approval voting. In: Proceedings of the 6th International Conference on Autonomous Agents and Multiagent Systems. pp. 1185-1187 (2007)

20. Lu, T., Boutilier, C.: Budgeted social choice: From consensus to personalized decision making. In: Proceedings of the Twenty-Second International Joint Conference on Artificial Intelligence. pp. 280-286 (2011)

21. Monroe, B.: Fully proportional representation. American Political Science Review 89(4), 925-940 (1995)

22. Niedermeier, R.: Invitation to Fixed-Parameter Algorithms. Oxford University Press (2006)

23. Ogryczak, W.: Multiple criteria linear programming model for portfolio selection. Annals of Operations Research 97(1-4), 143-162 (2000)

24. Procaccia, A., Rosenschein, J., Zohar, A.: On the complexity of achieving proportional representation. Social Choice and Welfare 30(3), 353-362 (2008) 
25. Skowron, P., Faliszewski, P., Lang, J.: Finding a collective set of items: From proportional multirepresentation to group recommendation. In: Proceedings of the 29th AAAI Conference on Artificial Intelligence. pp. 2131-2137 (2015)

26. Skowron, P., Faliszewski, P., Slinko, A.M.: Achieving fully proportional representation: Approximability results. Artificial Intelligence 222, 67-103 (2015)

27. Skowron, P., Yu, L., Faliszewski, P., Elkind, E.: The complexity of fully proportional representation for single-crossing electorates. Theoretical Computer Science 569, $43-57$ (2015)

28. Walsh, T.: Uncertainty in preference elicitation and aggregation. In: Proceedings of the 22nd National Conference on Artificial Intelligence. pp. 3-8 (2007)

29. Yager, R.R.: On ordered weighted averaging aggregation operators in multicriteria decisionmaking. Systems, Man and Cybernetics, IEEE Transactions on 18(1), 183-190 (1988)

30. Yager, R.R.: Constrained OWA aggregation. Fuzzy Sets and Systems 81(1), 89-101 (1996)

31. Yager, R.R., Kacprzyk, J.: The ordered weighted averaging operators: theory and applications. Springer Publishing Company, Incorporated (2012)

32. Yu, L., Chan, H., Elkind, E.: Multiwinner elections under preferences that are single-peaked on a tree. In: Proceedings of the 23rd International Joint Conference on Artificial Intelligence. pp. 425-431 (2013) 